\title{
A Theoretical Study on the Macrocyclic Strain of Zigzag Molecular Belts
}

\author{
Tan-Hao Shi ${ }^{\circledR}(\odot)$ \\ Shuo Tong ${ }^{\mathrm{a}}$ () \\ Lei Jiao ${ }^{\mathrm{b}, *}$ (1) \\ Mei-Xiang Wang ${ }^{\mathrm{a}, *}$ (iD \\ ${ }^{a}$ MOE Key Laboratory of Bioorganic Phosphorous and Chemical Biology, Department of Chemistry, \\ Tsinghua University, Beijing 100084, China \\ ${ }^{\text {b } C e n t e r ~ o f ~ B a s i c ~ M o l e c u l a r ~ S c i e n c e, ~ D e p a r t m e n t ~ o f ~ C h e m i s t r y, ~ T s i n g h u a ~ U n i v e r s i t y, ~ B e i j i n g ~ 100084, ~ C h i n a ~}$ \\ leijiao@mail.tsinghua.edu.cn, \\ wangmx@mail.tsinghua.edu.cn
}

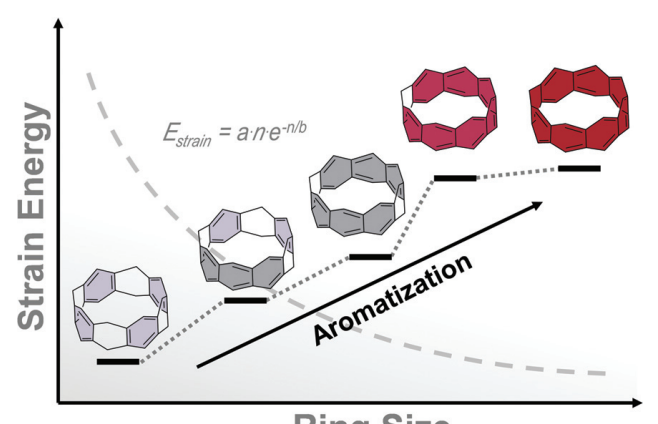

Received: 24.08.2020

Accepted after revision: 14.09 .2020

DOI: 10.1055/s-0040-1718934; Art ID: om-20-0026 sc

License terms: CC

(C) 2020. The Author(s). This is an open access article published by Thieme under the terms of the Creative Commons Attribution-NonDerivative-NonCommercial-License, permitting copying and reproduction so long as the original work is given appropriate credit. Contents may not be used for commercial purposes, or adapted, remixed, transformed or built upon. (https://creativecommons.org/licenses/by-nc-nd/4.0/).

Abstract Zigzag molecular belts have captured the imagination of scientists for over a half century because of their aesthetically appealing structures and tantalizing properties. One of the formidable challenges in synthesis is to circumvent the energy accumulated in the construction of strained structures. Reported herein is our theoretical study to quantify the molecular strain energies. A general exponential function equation $E_{\text {strain }}=a \cdot n \cdot e^{-n / b}$ was obtained to estimate strain energies of both conjugated and partially hydrogenated hydrocarbon belts and their heteroatom-embedded analogs. The deformation of aromatic rings from planarity was revealed to contribute dominantly to the high strain energies. The method enabled the convenient quantification of the energetics of aromatization processes from partially hydrogenated double-stranded macrocycles, and facilitated the design and optimization of practical routes to synthesize the longawaited zigzag molecular belts.

Key words zigzag hydrocarbon belts, belt[n]arenes, macrocycles, strain energy

\section{Introduction}

Zigzag hydrocarbon molecular belts such as fully conjugated belt $[n]$ arenes or cyclacenes $\mathbf{1}$ and their partially hydrogenated analogs including $\mathrm{H}_{n}$-belt $[n]$ arenes or collar[ $n$ ]arenes 2 and belt[ $n$ ]enes have been fascinating scientists for decades ${ }^{1-6}$ since they were proposed as imaginary molecules by Heilbronner ${ }^{7}$ in 1954 and as synthetic targets by Vögtle ${ }^{8}$ in 1983. Despite their aesthetically appealing structures, theoretically predicted tantalizing properties, ${ }^{9-15}$ and potential application as templates or seeds ${ }^{16}$ to grow structurally well-defined zigzag carbon nanotubes, synthesis of zigzag hydrocarbon belt molecules remains a formidable challenge. The molecular strain energy has been noted as one of the biggest hurdles in the synthetic process to construct double-stranded macrocycles. ${ }^{8,10,15,17}$ To circumvent the strain problem, Stoddart and coworkers ${ }^{18-20}$ developed an effective method featuring repetitive Diels-Alder reactions between precisely predesigned oxygen-bridged bisdienes and bisdienophiles with a rigid molecular curvature to construct kohnkene, a $\mathrm{H}_{20}$-belt[12]arene derivative densely functionalized with bridge-oxygen atoms on the outer surface. The same strategy has been used by others ${ }^{21-24}$ to construct partially hydrogenated belt $[n]$ arene derivatives. Attempts to obtain conjugated belt $[n]$ arenes were not successful. Very recently, we $e^{25-27}$ have discovered that resorcin[ $n]$ arenes $(n=4,6) 3$ are able to undergo the Fjord-stitching processes via multiple intramolecular alkylation and acylation reactions producing partially hydrogenated molecular belts such as $\mathrm{H}_{8}$-belt [8]arenes and $\mathrm{H}_{12}$-belt[12]arenes. Most remarkably, oxidative aromatization of a $\mathrm{H}_{8}$-belt[8]arene with 2,3dichloro-5,6-dicycno-1,4-benzoquinone (DDQ) affords a belt[8]arene- $\mathrm{DDQ}_{4}$ adduct which forms significantly the conjugated belt[8]arene under MALDI-MS conditions. ${ }^{25}$ Our interest ${ }^{25-28}$ in the facile construction of $\mathrm{H}_{n}$-belt $[n]$ arenes from monomacrocyclic rings and then their conversions to fully conjugated belt $[n]$ arenes led us to initiate the current project. We report herein our theoretical study on the strain energies (SE) and molecular structures of a series of diverse partially and fully conjugated zigzag hydrocarbon belts 1-2 and heteroatom-embedded analogs 4-6 (Figure 1). We will also report the energetics of the aromatization processes of $\mathrm{H}_{n}$-belt $[n]$ arenes. 


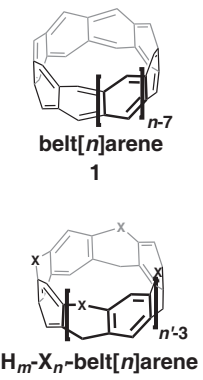

4

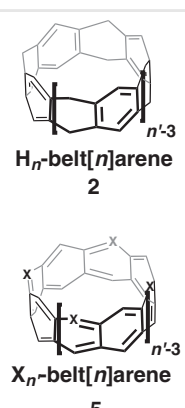

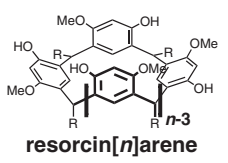

3

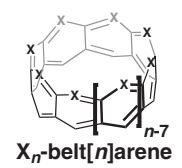

Figure 1 Structures of resorcin[n]arene and zigzag nanobelts $\left(n^{\prime}=n / 2\right)$.

\section{Results and Discussion}

The calculations were performed with the Gaussian 09 package. ${ }^{29}$ Geometry optimizations were carried out using the B3LYP $\mathrm{LP}^{30-32}$ functional with the 6-31G(d) basis set $^{33,34}$ in the gas phase. Vibrational frequency analysis was carried out at the same level to confirm the structure was a minimum. We commenced our study with the calculation of intrinsic strain energies of $\mathrm{H}_{n}$-belt $[n]$ arenes $\mathbf{2}$ as the reaction heat of the hypothetical homodesmotic reactions ${ }^{35,36}$ (Figure S1). The strain energies were found to decrease with the increase of the diameter of the belts. However, negative energy values were computed when the number of dihydronaphthalene repeating units exceeded eight. We noticed that Itami and coworkers have pointed out that a homodesmotic reaction might not be reliable to assess the strain energies of fully conjugated belt $n$ ]arenes. Alternatively, they identified a perfect linear correlation between the total electronic energy of a repeat unit $\left(\mathrm{C}_{4} \mathrm{H}_{2}\right)$ and $n^{-2}$ of belt $[n]$ arenes, and the strain energy for belt $[n]$ arenes was calculated to be $1365 / \mathrm{n} \mathrm{kcal} / \mathrm{mol}^{17,37}$ Therefore, we attempted to study the strain energies of the zigzag hydrocarbon belts of interest following this approach. Adapting the same treatment, we then plotted the total energy per number of repeat units $\left(E_{\text {unit }}=E_{\text {total }} / n^{\prime}\right)$ of $\mathrm{H}_{n^{-}}$ belt $\left[n\right.$ ]arenes $2\left(\mathrm{C}_{8} \mathrm{H}_{6}\right)_{n^{\prime}}\left(n^{\prime}=n / 2\right)$ as a function of $n^{\prime}, n^{\prime-1}$, and $n^{\prime-2}$ (Figures 2 and S2). As the results in Figures 2 and $\mathrm{S} 2$ indicate, $E_{\text {unit }}$ values correlated neither linearly with $n^{\prime-2}$ nor with $n^{\prime-1}$ and $n^{\prime}$. Interestingly, an exponential relationship was revealed between $E_{\text {unit }}$ and $n^{\prime}$ following the equation of $E_{\text {unit }}=464.95 \cdot e^{-n^{\prime} / 1.04}-193578.07$ $\left(R^{2}=0.9997\right)$ (Figure 2). According to the perfectly fitted equation, when $n^{\prime}$ approaches infinite, the limit of $E_{\text {unit }}$, the energy of the "unstrained" repeat unit, is -193578.07 $\mathrm{kcal} / \mathrm{mol}$. The strain energy of a repeat unit can be estimated as $464.95 \cdot e^{-n^{\prime} / 1.04} \mathrm{kcal} / \mathrm{mol}$. Therefore, the total strain energies of a number of $\mathrm{H}_{n}$-belt $[n$ ]arenes $\mathbf{2}$ were quantified from the equation: $E_{\text {strain }}=464.95 \cdot n^{\prime} \cdot e^{-n^{\prime} / 1.04}$, and are listed in Table S1.

Using the same density functional theory (DFT) calculations and data fitting methods, we obtained the similar

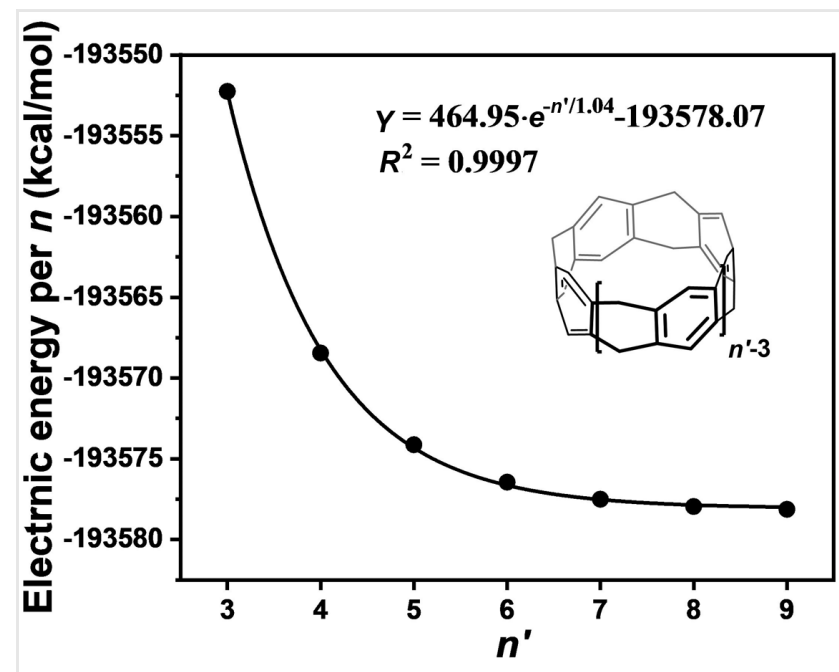

Figure 2 Correlation between total energy per number of repeat unit (Eunit) and $n^{\prime}$.

exponential equations (Figures S3-S7) between $E_{\text {unit }}$ and $n^{\prime}$ for all heteroatom-embedded partially hydrogenated $\mathrm{H}_{m}-\mathrm{X}_{n^{\prime}}$-belt [ $n$ ] arenes 4 which contain nitrogen, phosphorus, oxygen, sulfur atoms, or carbonyl linkages. Total strain energies of the corresponding belts were calculated (Table S1) and then plotted as a function of $n$ in Figure 3 .

As anticipated, the strain energies of partially hydrogenated belt $[n]$ arenes 2 and their heteroatom- $(X=N, P, O, S)$ or carbonyl-doped analogs 4 decreased with the increase of the belt sizes. It is noteworthy that the introduction of sulfur and phosphorus atoms made the resulting $\mathrm{H}_{m}-\mathrm{S}_{n^{\prime}}$-belt $[n$ ]arenes 4 $(\mathrm{X}=\mathrm{S})$ and $\mathrm{H}_{n}-\mathrm{P}_{n^{\prime}}$-belt $[n]$ arenes $4(\mathrm{X}=\mathrm{P})$ less strained than

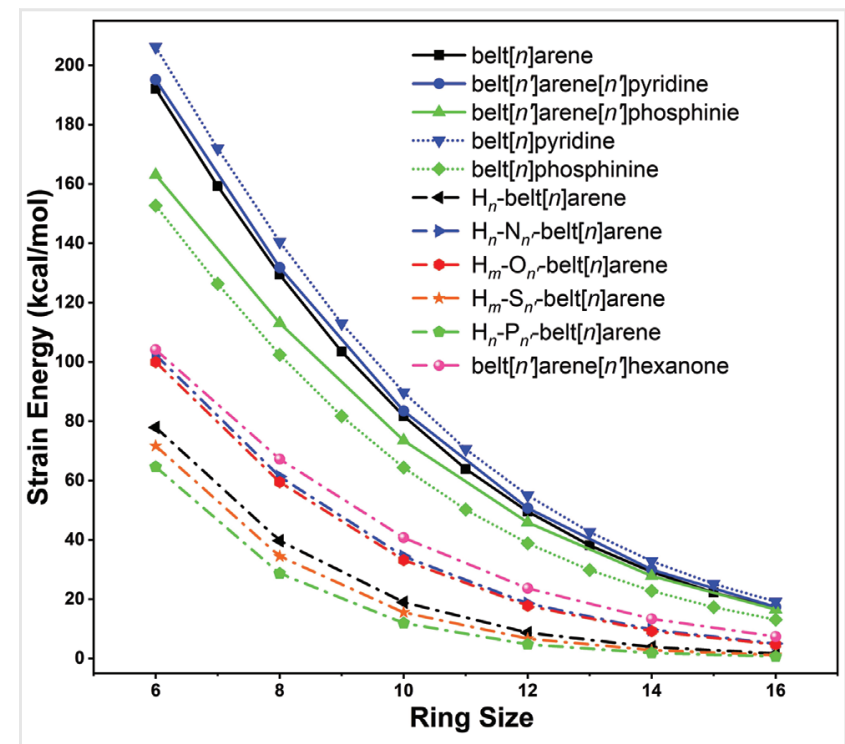

Figure 3 Strain energies for different $\mathrm{H}_{m}-\mathrm{X}_{n^{\prime}}$-belt[n]arenes and belt[n] (het)arenes $\left(n^{\prime}=n / 2\right)$. 
$\mathrm{H}_{n}$-belt $[n]$ arenes 2. However, the replacement of some methylenes in $\mathbf{2}$ with oxygen and nitrogen atoms resulted in the increase of considerable amounts of the strain energies, especially when the sizes of belt molecules are relatively small $\left(n=12\right.$ or $\left.n^{\prime}=6\right)$. Among all molecules 2 and 4 , the carbonylbridged belts have the highest energies, while the phosphinelinked ones are least strained.

The same theoretical method was then applied to quantify the strain energies of fully conjugated belt $[n]$ arenes $\mathbf{1}$ and their heteroatom-bearing analogs 5 and $\mathbf{6}$ (Figures S8-S15 and Tables S2 and S3). The strain energies were obtained readily from the equation $E_{\text {strain }}=n \cdot a \cdot e^{-n / b}$, in which $a$ and $b$ had different values depending on the structures ${ }^{17,37}$ (Supporting Information). The results plotted in Figure 3 indicated evidently that the strain energies of conjugated belts are substantially larger than those of their partially hydrogenated belt counterparts. For example, belt [10]arene appeared $62.8 \mathrm{kcal} / \mathrm{mol}$ more strained than $\mathrm{H}_{10^{-}}$ belt[10]arene. The smaller the belt size, the larger the difference between strain energies. The drastic increase of the strains may best explain the difficulty to fully aromatize partially hydrogenated belt[8]arenes. On the other hand, in comparison with $\mathbf{2}$ and $\mathbf{4}$, fully conjugated molecules 5 and $\mathbf{6}$ appeared much more sensitive toward the molecular size in terms of strain energy. This has been exemplified by an increase of strain energy of $79.8 \mathrm{kcal} / \mathrm{mol}$ from belt[12] arene to belt[8]arene, whereas the variation from $\mathrm{H}_{12}$-belt [12]arene to $\mathrm{H}_{8}$-belt[8]arene led to the gain of strain energy of only $31.0 \mathrm{kcal} / \mathrm{mol}$. In the case of heteroatom-embedded belt $[n]$ arenes $\mathbf{5}$ and $\mathbf{6}$, opposite effects of nitrogen and phosphorus on the strains were observed. While both belt $\left[n^{\prime}\right]$ arene $\left[n^{\prime}\right]$ pyridines $\mathbf{5}(\mathrm{X}=\mathrm{N})$ and belt $[n]$ pyridines $\mathbf{6}$ $(\mathrm{X}=\mathrm{N})$ were more strained than the corresponding belt $[n]$ arenes $\mathbf{1}$, incorporation of phosphorus atoms into belt $[n]$ arenes released the strain. It may also be worth addressing that the strain energies of lager conjugated belt $[n]$ arenes ( $n>11)$ are comparable with those of $\mathrm{H}_{8}$-belt[8]arenes, stable belt compounds which have been readily constructed, ${ }^{25}$ implying the macrocyclic strain would not be a barrier to impede the synthesis of larger belt[ $n]$ arenes $(n>11)$.

To gain insight into the macrocyclic strain, the geometric structures of zigzag belt molecules were scrutinized first. As illustrated in Figure $4, \mathrm{H}_{n}$-belt $[n]$ arenes adopted various symmetric polygonal prism structures depending on the number of repeat units. Replacement of methylene linkages by heteroatoms or carbonyl units on one edge resulted in the change of symmetric prisms into different truncated cones. Notably, all cyclohexa-1,4-diene subrings, six-membered heterocycles, and cyclohexa-2,5-dien-1-one subunits in belts formed a boat conformation. It should be addressed that the computed structures of $\mathrm{H}_{8}$-belt[8] arene and $\mathrm{H}_{12}$-belt[12]arene are supported by the X-ray single-crystal molecular structures of synthetic samples. ${ }^{25,26}$ Being different from partially

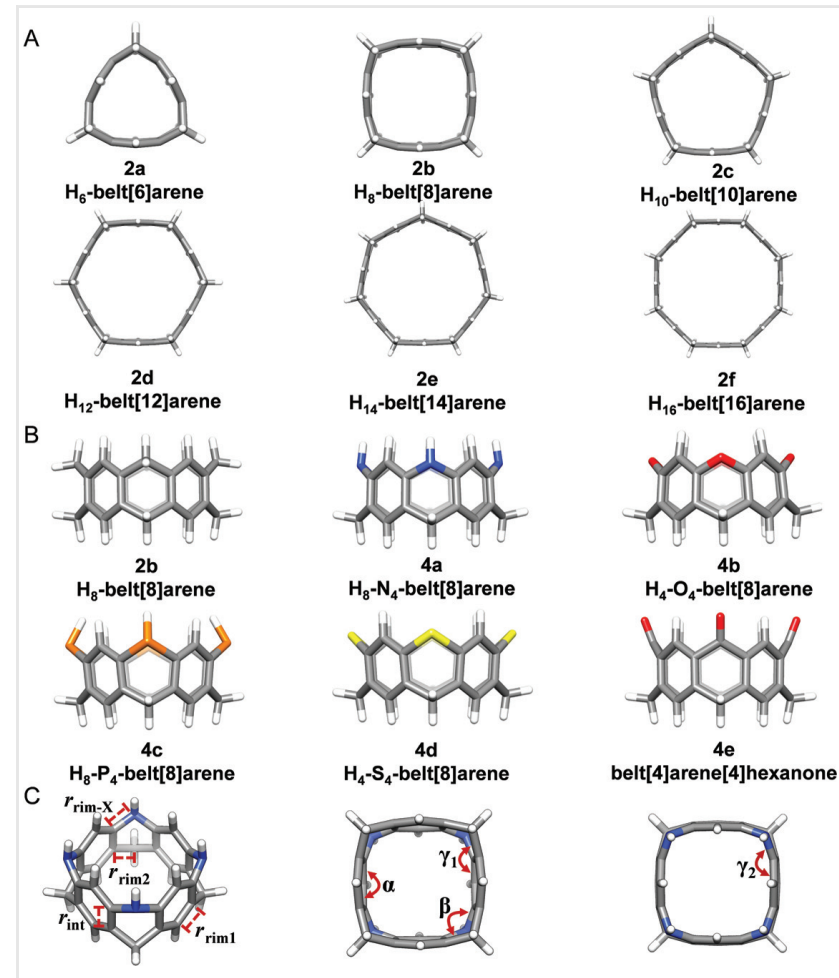

Figure 4 Optimized structures of $\mathrm{H}_{n}$-belt $[n]$ arenes with top view (A) and of $\mathrm{H}_{8}-\mathrm{X}_{4}$-belt[8]arenes with side view (B). Bond lengths $r_{\text {int }}, r_{\text {rim }}$, $r_{\text {rim } 1}, r_{\text {rim2 } 2}$, and $r_{\text {rim- }}$, and dihedral angles $\alpha, \beta, \gamma_{1}$, and $\gamma_{2}$ are defined (C). In the case of $\mathrm{H}_{n}$-belt[n]arenes, $\mathrm{Y}_{1}=\mathrm{Y}_{2}$.

hydrogenated belt $[n]$ arenes, conjugated zigzag hydrocarbon belts adopt nearly cylindrical structures (Figure 5). Transformations from symmetric cylinders to various truncated cones were observed when different heteroatoms were introduced into one of the edges of the belts. In the case of belt[8]pyridine, for instance, the diameter of the nitrogen atom-doped edge is shorter than that of all-carbon edge. Belt [8]phosphinine, on the other hand, gives a truncated cone cavity in which the circumference of the P-bearing rim is larger than that of all-carbon rim (Figure 5).

To shed further light on the origin of the strain of zigzag belts, structural parameters such as dihedral angles and bond lengths of optimized structures were defined (Figures 4, 5, and S20) and plotted as a function of belt size (Figures 6 and S21-S29). Figures 6A and S21A-S25A show that there is a very small bond length alternation between $r_{\text {int }}$ and $r_{\text {rim }}$ which is size-independent, indicating the presence of characteristic benzene subunits within all partially hydrogenated belt $[n]$ (hetero)arenes. The rapid increase of dihedral angles of $\alpha, \gamma_{1}$, and $\gamma_{2}\left(\gamma_{1}\right.$ equals $\gamma_{2}$ in the case of $H_{n}$-belt $[n]$ arenes) to approach the ideal angles of a planar conformation of the subrings from $\mathrm{H}_{6}$-belt[6]arene or $\mathrm{H}_{6}-\mathrm{X}_{3}$-belt[6]arenes to $\mathrm{H}_{18}$-belt[18]arene or $\mathrm{X}_{18}-\mathrm{X}_{9}$-belt[6]arenes, which are depicted clearly in Figures 6B and S21B-S25B, suggested 


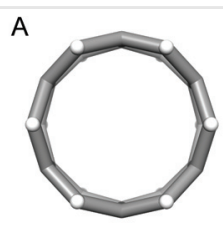

1a

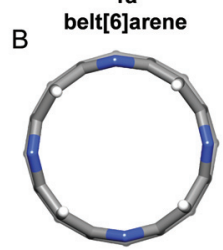

$5 \mathbf{a}$
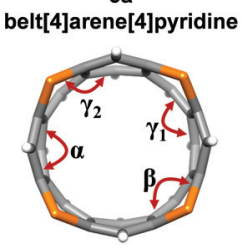

$5 b$

belt[4]arene[4]phosphinie
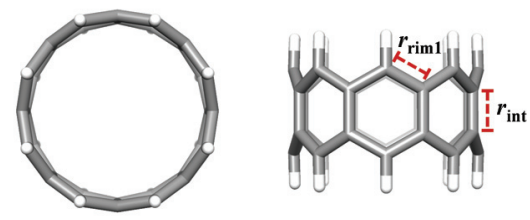

$1 b$ belt[8]arene
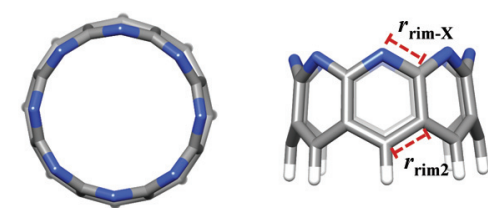

$6 a$

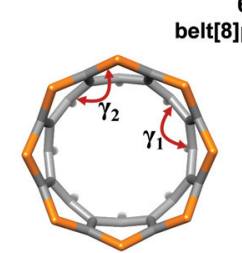

belt[8]pyridine

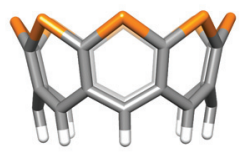

6b

belt[8]phosphinine
Figure 5 Optimized molecular structures of (A) belt $[n]$ arenes $(n=6,8)$ and (B) $\mathrm{N}$ - and $\mathrm{P}$-embedded belt[8](het)arenes with top and side views. Bond lengths $r_{\text {int }}, r_{\text {rim } 1}, r_{\text {rim2 } 2}$, and $r_{\text {rim-x }}$, and dihedral angles $\alpha, \beta, \gamma_{1}$, and $\mathrm{V}_{2}$ are defined (C).
A

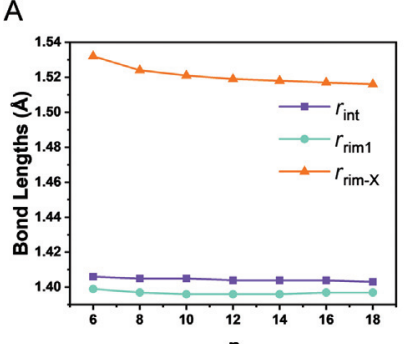

C

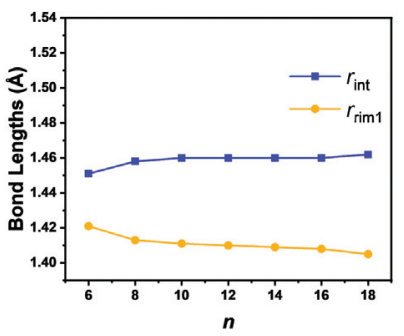

B

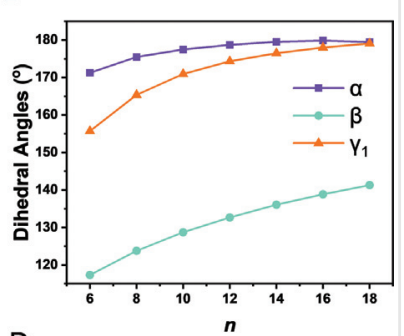

D

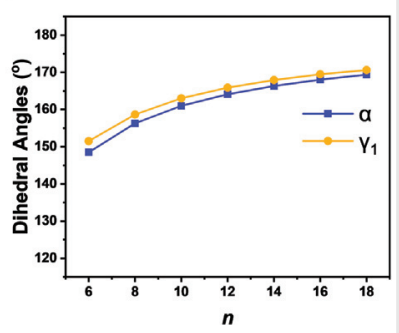

Figure 6 Bond lengths ( $A$ and $C$ ) and dihedral angles ( $B$ and $D$ ) of $\mathrm{H}_{n}$-belt [n] arenes $(A$ and $B)$ and belt $n$ ] arenes $(C$ and $D)$ as a function of belt sizes.

that the high strain energies of smaller partially hydrogenated belt $[n]$ arenes were mainly contributed from the deformation of benzene subunits and, particularly, from the deviation of methylenes and heteroatoms from the plane of their adjacent benzene rings. It is also interesting to note that, with the increase of belt sizes, the $\mathrm{C}-\mathrm{C}$ and $\mathrm{C}-\mathrm{S}, \mathrm{C}-\mathrm{P}, \mathrm{C}-\mathrm{C}=\mathrm{O}$ single bond lengths $\left(r_{\text {rim-X }}\right)$ decrease slightly while the $\mathrm{C}-\mathrm{N}$ and $\mathrm{C}-\mathrm{O}$ bonds are shortened considerably. For example, $\mathrm{H}_{6}-\mathrm{N}_{3}$-belt [6] arene and $\mathrm{H}_{3}-\mathrm{O}_{3}$-belt[6]arene give the $\mathrm{C}-\mathrm{N}$ and $\mathrm{C}-\mathrm{O}$ bond length $\left(r_{\text {rim-X }}\right)$ of $1.450 \AA$ And $1.414 \AA$, respectively. In the case of $\mathrm{H}_{18^{-}}$ $\mathrm{N}_{9}$-belt[18]arene and $\mathrm{H}_{9}-\mathrm{O}_{9}$-belt[18]arene, however, much shorter $\mathrm{C}-\mathrm{N}$ bond (1.409 $\AA$ ) and $\mathrm{C}-\mathrm{O}$ bond (1.384 $\AA$ ) are computed. The outcomes are the indication of the formation of a stronger conjugation of the lone-pair electrons on nitrogen and oxygen with their neighboring benzene rings in larger molecular belts. In comparison to the $\mathrm{C}-\mathrm{C}$ bond $\left(r_{\text {rim2 } 2}\right)$, the long $\mathrm{C}-\mathrm{P}$ and $\mathrm{C}-\mathrm{S}$ bonds $\left(r_{\text {rim-X }}\right)$, owing to the large atomic radius of the corresponding phosphorous and sulfur atoms, result in the expansion of the circumferences of heteroatomembedded rims, leading to the formation of truncated cones.

As shown by the plots in Figures $6 \mathrm{C}$ and S26-S29, comparing partially reduced $\mathrm{H}_{n}$-belt $[n]$ arenes and heteroatom-doped analogs, the disparity between $r_{\text {int }}$ and $r_{\text {rim }}$ is larger in belt[ $n$ ]arenes $\mathbf{1}$ and belt[ $n]$ (het)arenes $\mathbf{5}$ and $\mathbf{6}$. The elongated inter C-C bonds $\left(r_{\text {int }}>1.451 \AA\right.$ A $\left.n>8\right)$ implied a weaker delocalization of electrons between two conjugated layers. Dihedral angles $\alpha, \beta, \gamma_{1}$, and $\gamma_{2}$ within conjugated belts (Figures 5 and S20) are significantly smaller than those in the corresponding partially hydrogenated belt analogs (Figure 6B, D). Especially, there is noticeable deformation of six-membered rings from their planarity in smaller zigzag belts $(n<10)$. It is obviously the forced bending of planar segments that contributes as the dominant factors to build up macrocyclic strain. It should be mentioned that fully conjugated belt molecules such as belt $[n]$ arenes and belt $[n]$ (het)arenes were also computed using the unrestricted broken spin-symmetry DFT method ${ }^{13}$ (Supporting Information). Calculations showed that belt $[n]$ arenes, belt $\left[n^{\prime}\right]$ arene $\left[n^{\prime}\right]$ pyridines, and belt $[n]$ pyridines have open-shell ground states. However, no considerable difference in molecular geometries was evident. The strain energies were obtained following the same form of exponential equation $\left(E_{\text {strain }}=n \cdot a \cdot e^{-n / b}\right)$ albeit the strain energies were slightly lower using the UB3LYP method (Table S3).

Finally, the established computational method was utilized to study the energetics of the aromatization of $\mathrm{H}_{8}$-belt[8]arene to form belt[8]arene, which was observed experimentally by means of MALDI mass spectrometry. ${ }^{25}$ Since the complete aromatization of $\mathrm{H}_{8}$-belt[8]arene proceeds probably through two distinct step-by-step routes, DFT calculations of partially hydrogenated belt $[n]$ arenes which are composed of different repeat units (Figure 7) were executed (Figures S16-S19). The similar forms of the exponential function equation $E_{\text {strain }}=n \cdot a \cdot e^{-n / b}(a$ and $b$ values were determined by the structures) to estimate strain energies of $\mathrm{H}_{6 n}$-belt[8n]arenes 7, $\mathrm{H}_{4 n}$-belt[4n]arenes $\mathbf{8}$ and $\mathbf{9}$, and $\mathrm{H}_{2 n}$-belt[2n]belts $\mathbf{1 0}$ were computed and are listed in Figure 7. Belt $\mathbf{8}$ is more stable than $\mathbf{9}$, as the largest conjugated subunit in $\mathbf{8}$ is anthracene whereas $\mathbf{9}$ contains 


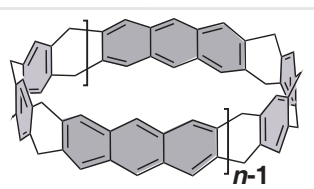

$\mathrm{H}_{6 n}$-belt[8n]arene

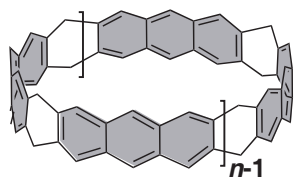

$\mathrm{H}_{4 n}$-belt[4n]arene

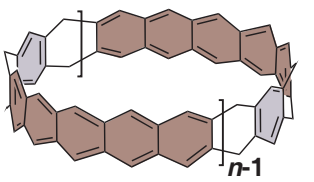

$\mathrm{H}_{4 n}$-belt[4n]arene

$E_{\text {strain }}=1051.64 \cdot n \cdot \mathrm{e}^{-n / 0.43}$

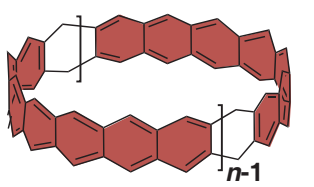

$\mathrm{H}_{2}$-belt[2n]arene

$E_{\text {strain }}=843.45 \cdot n \cdot \mathrm{e}^{-n / 0.53}$
$E_{\text {strain }}=1298.17 \cdot n \cdot \mathrm{e}^{-n / 0.34}$

$E_{\text {strain }}=791.61 \cdot n \cdot \mathrm{e}^{-n / 0.46}$
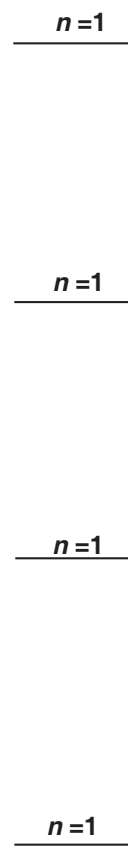

Figure 7 Strain energies of different partially hydrogenated beltarenes.

pentacene. The calculations demonstrated quantitatively an uphill process to aromatize $\mathrm{H}_{8}$-belt[8]arene to form belt[8] arene via most likely intermediates $7(\mathrm{SE}=68.5 \mathrm{kcal} / \mathrm{mol}), 8$ $(\mathrm{SE}=90.0 \mathrm{kcal} / \mathrm{mol})$, and $\mathbf{1 0}(\mathrm{SE}=127.8 \mathrm{kcal} / \mathrm{mol})$ (Figure 7$)$. It is worth noting that, besides strain, the fully conjugated belt [ $n$ ]arenes are probably more prone to cycloaddition with oxygen or dimerization than the linear polyacenes, such as anthracene, [5]acene, or [7] acene (Figures S29 and S30). These may also jeopardize the isolation of belt[ $n$ ] arenes.

\section{Conclusions}

Our theoretical study has provided a general exponential function equation $E_{\text {strain }}=a \cdot n \cdot e^{-n / b}(a$ and $b$ values are varied depending on the molecular structures) to estimate strain energies of both conjugated and partially hydrogenated hydrocarbon belts and their heteroatom-embedded analogs. We have also revealed that the forced deformation of aromatic rings from planarity contributes dominantly to the high strain of belt molecules. The method enables the convenient quantification of the energetics of aromatization processes from partially hydrogenated double-stranded macrocycles, facilitating the design and optimization of practical routes to synthesize the long-awaited zigzag molecular belts.

\section{Funding Information}

We thank the National Science Foundation of China (grant No. 21732004 and 21821001) and Tsinghua University Initiative Scientific Research Program for financial support.

\section{Supporting Information}

Supporting information for this article is available online at http://doi.org/10.1055/s-0040-1718934. It shows detailed calculation results.

\section{Primary Data}

Primary data including the Cartesian coordinates for this article are available online at: http://doi.org/10.1055/ s-0040-1718934 and can be cited using the following DOI: $10.4125 /$ pd0123th.

\section{References and Notes}

(1) Shi, T-H.; Wang, M-X. CCS Chem. 2020, 2, 916.

(2) Cheung, K. Y.; Segawa, Y.; Itami, K. Chemistry 2020. Doi: 10.1002/ chem. 202002316.

(3) Eisenberg, D.; Shenhar, R.; Rabinovitz, M. Chem. Soc. Rev. 2010, 39, 2879.

(4) Gleiter, R.; Esser, B.; Kornmayer, S. C. Acc. Chem. Res. 2009, 42, 1108.

(5) Esser, B.; Bandyopadhyay, A.; Rominger, F.; Gleiter, R. Chemistry 2009, 15, 3368.

(6) Tahara, K.; Tobe, Y. Chem. Rev. 2006, 106, 5274.

(7) Heilbronner, E. Helv. Chim. Acta 1954, 37, 921.

(8) Vögtle, F. Top. Curr. Chem. 1983, 115, 157.

(9) Kivelson, S.; Chapman, O. L. Phys. Rev. B: Condens. Matter 1983, $28,7236$.

(10) Choi, H. S.; Kim, K. S. Angew. Chem. Int. Ed. 1999, 38, 2256.

(11) Wu, C. S.; Lee, P. Y.; Chai, J. D. Sci. Rep. 2016, 6, 37249.

(12) Sadowsky, D.; McNeill, K.; Cramer, C. J. Faraday Discuss. 2010, 145, 507.

(13) Chen, Z.; Jiang, D-E.; Lu, X.; Bettinger, H. F.; Dai, S.; Schleyer, Pv.; Houk, K. N. Org. Lett. 2007, 9, 5449.

(14) Battaglia, S.; Faginas-Lago, N.; Andrae, D.; Evangelisti, S.; Leininger, T. J. Phys. Chem. A 2017, 121, 3746.

(15) San-Fabián, E.; Pérez-Guardiola, A.; Moral, M.; Pérez-Jimenez, A. J.; Sancho-García, J. C. Advanced Magnetic and Optical Materials. Tiwari, A.; Iyer, P. K.; Kumar, V.; Swart, H., Eds. Scrivener Publishing LLC: Beverly, 2017.

(16) Omachi, H.; Nakayama, T.; Takahashi, E.; Segawa, Y.; Itami, K. Nat. Chem. 2013, 5, 572.

(17) Segawa, Y.; Yagi, A.; Ito, H.; Itami, K. Org. Lett. 2016, 18, 1430.

(18) Kohnke, F. H.; Slawin, A. M. Z.; Stoddart, J. F.; Williams, D. J. Angew. Chem. Int. Ed. 1987, 26, 892.

(19) Ashton, P. R.; Isaacs, N. S.; Kohnke, F. H.; Slawin, A. M.Z.; Spencer, C. M.; Stoddart,J. F.; Williams, D.J.Angew. Chem. Int.Ed. 1988, 27, 966.

(20) Ashton, P. R.; Brown, G. R.; Isaacs, N. S.; Giuffrida, D.; Kohnke, F. H.; Mathias, J. P.; Slawin, A. M. Z.; Smith, D. R.; Stoddart, J. F.; Williams, D. J. J. Am. Chem. Soc. 1992, 114, 6330. 
(21) Godt, A.; Enkelmann, V.; Schlüter, A-D. Angew. Chem. Int. Ed. 1989, 28, 1680.

(22) Cory, R. M.; McPhail, C. L.; Dikmans, A. J.; Vittal, J. J. Tetrahedron Lett. 1996, 37, 1983.

(23) Schulz, F.; Garcia, F.; Kaiser, K.; Pérez, D.; Guitián, E.; Gross, L.; Peña, D. Angew. Chem. Int. Ed. 2019, 58, 9038.

(24) Chen, H.; Gui, S.; Zhang, Y.; Liu, Z.; Miao, Q. CCSChem. 2020, 2, 613.

(25) Shi, T-H.; Guo, Q-H.; Tong, S.; Wang, M-X.J. Am. Chem. Soc. 2020, $142,4576$.

(26) Shi, T-H.; Tong, S.; Wang, M-X.Angew. Chem. Int.Ed. 2020, 59, 7700.

(27) Zhang, Y.; Tong, S. Angew. Chem. Int. Ed. 2020. Doi: 10.1002/ anie.202006231.

(28) Zhang, Q.; Zhang, Y-E.; Tong, S.; Wang, M-X. J. Am. Chem. Soc. 2020, 142, 1196.
(29) Frisch, M. J., et al. Gaussian 09, Rev. D.01. Gaussian, Inc.: Wallingford, 2010.

(30) Becke, A. D. Phys. Rev. A 1988, 38, 3098.

(31) Becke, A. D. J. Chem. Phys. 1993, 98, 5648.

(32) Yang, W.; Parr, R. G. Phys. Rev. B: Condens. Matter 1988, 37, 785.

(33) Ditchfield, R.; Hehre, W. J.; Pople, J. A. J. Chem. Phys. 1971, $54,72$.

(34) Hariharan, P. C.; Pople, J. A. Theor. Chim. Acta 1973, 28, 213.

(35) George, P.; Trachtman, M.; Bock, C. W.; Brett, A. M. Tetrahedron 1976, 32, 317.

(36) Minkin, V. I. Pure Appl. Chem. 1999, 71, 1919.

(37) Different strain energies of belt[ $n$ ]arenes were reported by the authors in the text and in the Supporting Information of the paper (cf. Ref. 17). We checked the data and found that reported in the Supporting Information is correct. 